\title{
Metastasis of renal clear-cell carcinoma to the oral mucosa, an atypical location
}

\author{
Óscar Maestre-Rodríguez ${ }^{1,3}$, Raúl González-García ${ }^{2}$, Jesús Mateo-Arias ${ }^{2}$, Carlos Moreno-García ${ }^{3}$, Hermi- \\ nia Serrano-Gil ${ }^{3}$, Laura Villanueva-Alcojol ${ }^{3}$, Ana $\mathrm{M}^{\mathrm{a}} \mathrm{Campos}_{\mathrm{C}}$ Orellana ${ }^{4}$, Florencio Monje-Gil ${ }^{2,5}$ \\ ${ }^{1}$ Doctor, specialist in Stomatology \\ ${ }^{2}$ Oral and Maxillofacial Surgeon. Department of Oral and Maxillofacial Surgery, Infanta Cristina University Hospital. Badajoz \\ ${ }^{3}$ Resident Physician. Department of Oral and Maxillofacial Surgery, Infanta Cristina University Hospital. Badajoz \\ ${ }^{4}$ Doctor, specialist in Pathological Anatomy. Department of Pathological Anatomy, Infanta Cristina University Hospital. Badajoz \\ ${ }^{5}$ Department Chair. Department of Oral and Maxillofacial Surgery, Infanta Cristina University Hospital. Badajoz
}

Correspondence:

Servicio de Cirugía Oral y Maxilofacial

Hospital Universitario Infanta Cristina.

Avenida de Elvas $s / n$.

06080.Badajoz, Spain,

oscar@clinicamaestre.com

Received: $22 / 11 / 2008$

Accepted: $15 / 08 / 2009$

\begin{abstract}
Maestre-Rodríguez O, González-García R, Mateo-Arias J, MorenoGarcía C, Serrano-Gil H, Villanueva-Alcojol L, Campos-de Orellana A, Monje-Gil F. Metastasis of renal clear-cell carcinoma to the oral mucosa, an atypical location. Med Oral Patol Oral Cir Bucal. 2009 Nov 1;14 (11):e601-4.

http://www.medicinaoral.com/medoralfree01/v14i11/medoralv14i11p601.pdf
\end{abstract}

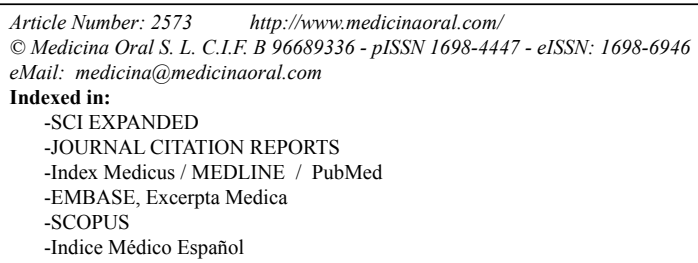

\begin{abstract}
The majority of cases of metastatic tumors involve the mandible and some the maxilla but they are considerably less common in intraoral soft tissues. In addition, the primary tumor is known in the majority of cases; although in onethird of such cases, metastasis is the first clinical manifestation. The most common primary tumors metastasizing to the mouth are lung carcinoma in men and breast carcinoma in women. An oral metastasis implies a serious prognosis, as in the majority of patients there is multiple organ involvement at the time of diagnosis. We present the case of a 52-year old patient with renal pathology who came to the emergency room due to a rapidly increasing gingival tumor. With the provisional clinical diagnosis of a pyogenic granuloma,the tumor was excised. Subsequent anatomopathological analysis revealed a tumor metastasis compatible with clear-cell carcinoma, and its renal origin was confirmed by means of immunohistochemical techniques.
\end{abstract}

Key words: Gingival metastasis, gingiva, renal carcinoma.

\section{Introduction}

Metastatic tumors in the oral cavity are very uncommon and represent approximately $1 \%$ of all cases of neoplasms in the oral cavity. In the majority of cases, the primary tumor is known; although in one-third of such cases, metastasis is the first clinical manifestation. The most common primary tumors are lung carcinoma in men and breast carcinoma in women.

Renal carcinoma is the third most common infraclavicular neoplasm that metastasizes to the oral cavity, following that of lung and breast carcinoma (1). Renal carcinoma typically affects men between the ages of 30 and 60 years old, and may metastasize to any part of the body (2), with a $15 \%$ risk of metastasis to the head and neck when the disease is disseminated and a $1 \%$ risk when it is not. Metastatic cervical adenopathy is reported to be the most common. Other forms that may be present in the head and neck are epistaxis, anosmia, facial pain, nasal obstruction and dyplopia. 
We present a case of clear-cell renal carcinoma metastasis to the oral cavity that began clinically as a gingival granuloma in a patient with radicular remains, polycaries and advanced periodontal disease.

\section{Case Report}

A 52-year old male is referred to the emergency room for gingivorrhagia, difficulty chewing and swallowing movements secondary to a $4 \times 4 \mathrm{~cm}$ granulomatous gingival lesion at a previous site in the superior maxilla (Fig. 1A). Physical exploration reveals a friable, polypoid tumor with a wide implantation base (Fig. 2B) that includes incisors 12,11 and 21, with significant mobility in these teeth. An ortho-pantomogram (OPG) reveals partial edentulism with advanced periodontal disease; significant alterations to the bone are not observed. With the clinical judgment of pyogenic granuloma vs. neoplasm, the patient is admitted and a complete exeresis biopsy of the lesion is performed in order to control the bleeding; incisors 12, 11 and 21 are also extracted (Fig. 1C and D).

During admission, the patient asks about pain and inflammation that he has been experiencing in the fourth finger of the left hand over an approximate period of 6 months. A postero-anterior and lateral X-ray (Fig. 2) reveal the disappearance of the distal phalanx and

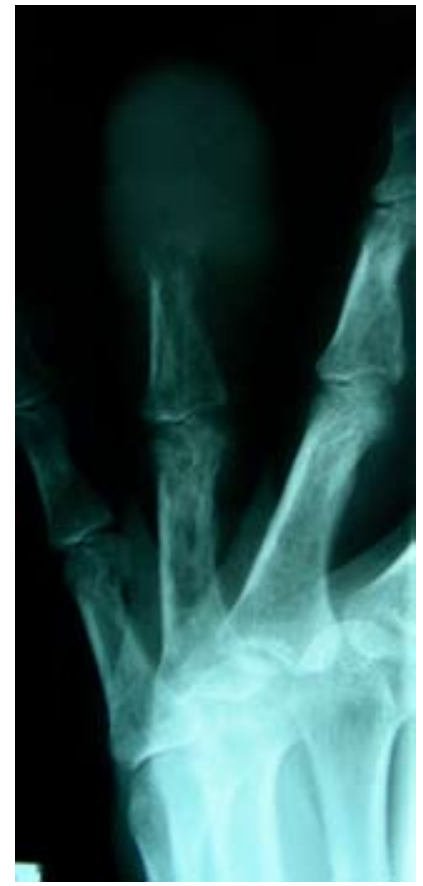

Fig.2 Simple postero-anterior Xray of the hand.

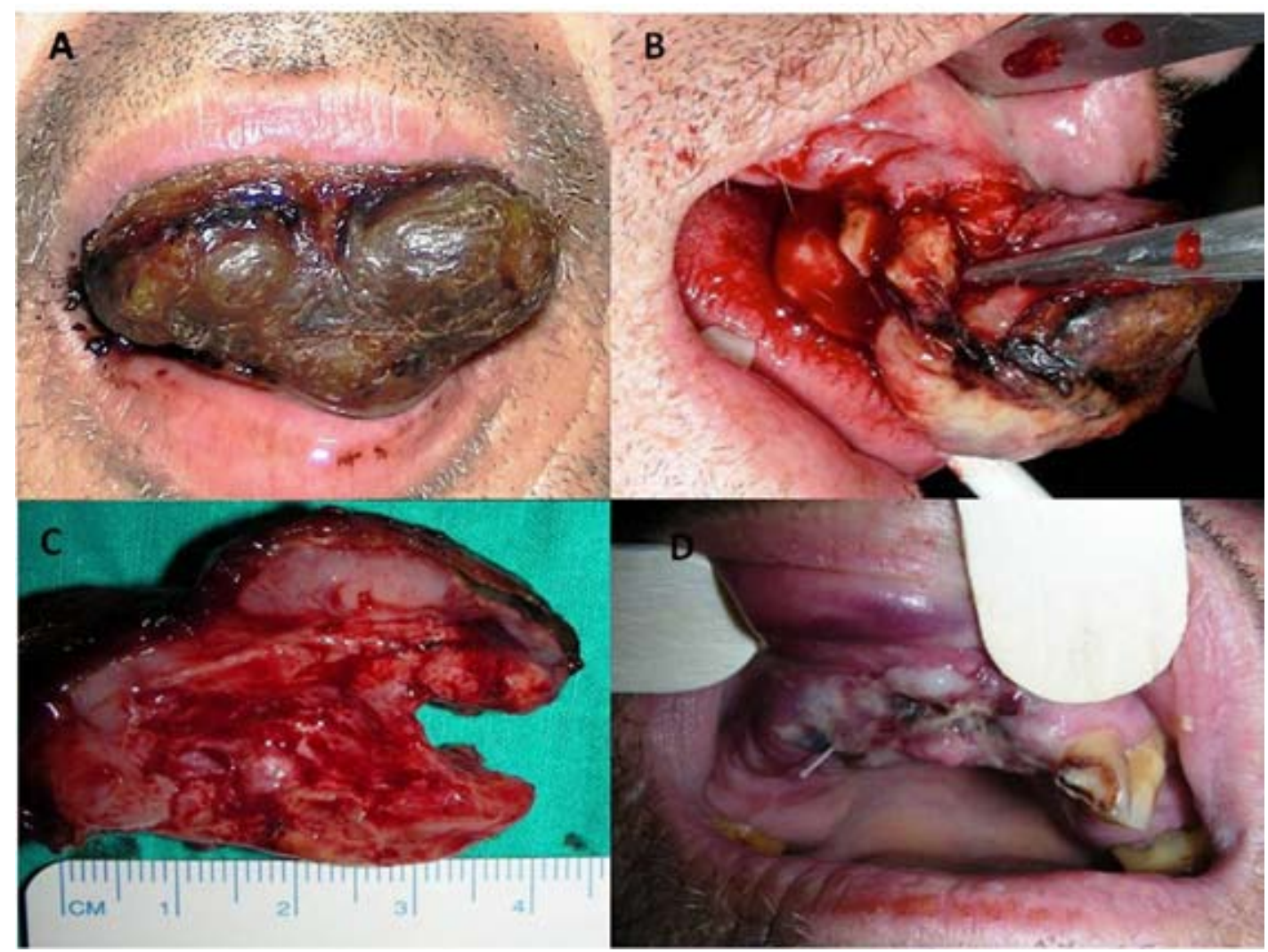

Fig.1 A- Gingival tumor, B- Exeresis, C- Resected lesion, D- 7 days post-op. 


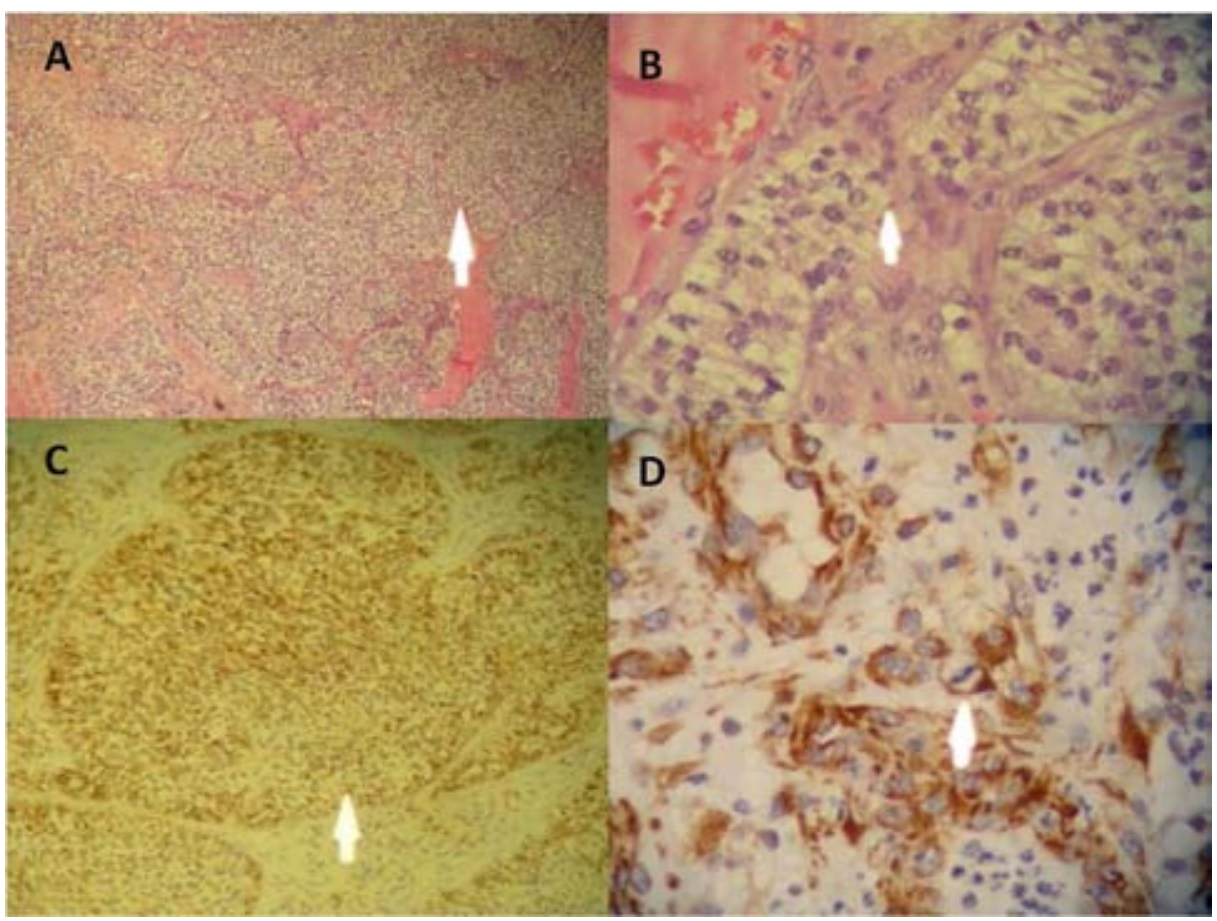

Fig. 3. A and B- Hematoxylin and Eosin Staining C and D - Staining with immunohistochemical techniques.

involvement of the middle phalanx, leading to a subsequent clinical diagnosis of osteomyelitis vs. metastasis; therefore, partial amputation of the fourth finger is scheduled.

The anatomopathological report confirms the neoplastic origin of the gingival lesion with images compatible with clear-cell carcinoma.

During the extensive study and search for a primary tumor and while waiting to receive the definitive anatomopathological study, the patient dies due to metastasis involving multiple organs. The subsequent pathological study confirms the metastatic origin of the gingival lesion, with images compatible with clear-cell carcinoma (Fig. 3A and B). The use of immunohistochemical techniques confirms the renal origin (Fig. $3 \mathrm{C}$ and D).

\section{Discussion}

The frequency of metastatic tumors in the oral cavity is less than $1 \%$ (3). The tumors of origin are, in decreasing order of frequency, breast, lung, kidney and prostate carcinoma; however, if we consider only the cases that metastasize to soft tissue, lung carcinoma is the most frequent primary tumor (4).

Metastases involving the gingiva usually appear in the form of a polypoid tumor, requiring the use of immunohistochemical techniques during the anatomopathological study in order to confirm a primary or metastatic origin of the neoplasm. The differential diagnosis must be made with the giant cell granuloma, haemangioma and fibroma, depending on the final diagnosis and course of treatment planned following the result of the biopsy.

The majority of metastatic intraoral tumors appear in the context of dissemination to multiple organs, as occurred in this case; however in $30 \%$ of the cases, oral metastasis may be the initial clinical data leading to the diagnosis of a primary tumor, and therefore in these cases, it is important to carry out an early Body-Scan and / or a PET-Scan.

In the oral cavity, the majority of metastases are to the bones, especially involving the jaw. Only in 33\% of the cases are they seated in soft tissues, mainly the gingiva and tongue. In our case, it was exclusively the gingiva that was involved. Several authors (5-6) associate the presence of a metastasis in soft tissue with a bad prognosis, due to the rapid growth and dissemination of the primary tumor to multiple organs.

As far as that pertaining to the pathogenesis of intraoral metastasis, Hirshberg (7) suggests that gingival inflammation plays an important role in attracting metastatic cells to the gingiva. In these cases, metastasis to the lungs is usually already present, although it is described as metastasis directly to the oral cavity through the vertebral venous plexus (8).

The differential diagnosis of a malignant intraoral neoplasm should include the metastatic tumor, although this probability is low, and even more so if we know that there has been previous existence of a tumor. Otherwise, this could lead to the diagnosis of an unknown 
primary tumor; although, there are cases of metastases described as occurring from 17 to 20 years after having treated the primary tumor (9). In the case of metastatic tumors for which the primary tumor is unknown, we advocate performing a Pet-scan if the results of the Body-Scan are negative.

The treatment will depend on many factors; however in cases of metastasis to soft tissue, complete excision is advisable regardless of the degree of dissemination (1-10). If it involves the bone and is the only location where it is seated, surgical resection is advised if possible, but if the extension study reveals evidence of the spread of the primary tumor or that the metastasis cannot be resected, palliative treatment is recommended. Although renal clear-cell carcinoma is a radio-resistant tumor, radiotherapy treatment may be useful in the treatment of the metastatic disease (11). Chemotherapy treatment (interleukin-2, interferon alpha and 5-fluorouracil) may be useful in cases of residual disease after resection, although the partial response is less than $25 \%$ (1).

In summary, an oral metastasis implies a serious prognosis, as most patients present multiple organ involvement at the time of diagnosis, with the average survival time being a few weeks or months following the presentation of gingival metastasis.

\section{References}

1. Pritchyk KM, Schiff BA, Newkirk KA, Krowiak E, Deeb ZE. Metastatic renal cell carcinoma to the head and neck. Laryngoscope. 2002;112:1598-602.

2. Cheng ET, Greene D, Koch RJ. Metastatic renal cell carcinoma to the nose. Otolaryngol Head Neck Surg. 2000;122:464.

3. Van der Waal RI, Buter J, Van der Waal I. Oral metastases: report of 24 cases. Br J Oral Maxillofac Surg. 2003;41:3-6.

4. Hirshberg A, Buchner A. Metastatic tumours to the oral region. An overview. Eur J Cancer B Oral Oncol. 1995;31B:355-60.

5. Ellis GL, Jensen JL, Reingold IM, Barr RJ. Malignant neoplasms metastatic to gingivae. Oral Surg Oral Med Oral Pathol. 1977;44:23845.

6. Peris K, Cerroni L, Paoloni M, Margiotta V, Chimenti S. Gingival metastasis as first sign of an undifferentiated carcinoma of the lung. J Dermatol Surg Oncol. 1994;20:407-9.

7. Hirshberg A, Leibovich P, Buchner A. Metastases to the oral mucosa: analysis of 157 cases. J Oral Pathol Med. 1993;22:385-90.

8. Miyamoto R, Helmus C. Hypernephroma metastatic to the head and neck. Laryngoscope. 1973;83:898-905.

9. Coppa GF, Oszczakiewicz M. Parotid gland metastasis from renal carcinoma. Int Surg. 1990;75:198-202.

10. Alvarez-Alvarez C, Iglesias-Rodríguez B, Pazo-Irazu S, Delgado-Sánchez-Gracián C. Colonic adenocarcinoma with metastasis to the gingiva. Med Oral Patol Oral Cir Bucal. 2006;11:E85-7.

11. Narea-Matamala G, Fernández-Toro Mde L, Villalabeitía-Ugarte E, Landaeta-Mendoza M, Rojas-Alcayaga G. Oral metastasis of renal cell carcinoma, presentation of a case. Med Oral Patol Oral Cir Bucal. 2008;13:E742-4. 